\section{The Effect of Attachment on Post-psychotic Trauma in First Episode Psychosis}

\section{Abstract}

Objective: To further understand the role that attachment plays in post-psychotic trauma of the first episode of psychosis.

Design: A systematic review was conducted, reviewing and appraising the current literature of trauma, attachment, and first episode psychosis in clinical samples.

Methods: Research utilizing East Carolina University's One Search, a database of all available articles to the university using the keywords trauma, attachment, first episode psychosis. Twenty-eight articles were identified that studied attachment's effect on the trauma of first episode psychosis.

Results: The authors identified and themed articles $(n=28)$. The themes included early trauma, mentalization, symptomatology, attachment type, anxiety disorder, coping, and adjustment/recovery. There were also several that were outliers. The themes amongst the outliers were mindfulness, symptomatology, metacognition, recovery, coping, and personality disorder that appeared to be unrelated to the other articles in the systematic review.

Conclusion: Attachment appears to have a correlation with post-psychotic recovery and trauma. Almost $50 \%$ of the articles $(n=12)$ across multiple disciplines appear to support the same thesis statement that individuals with insecure type attachment (avoidant or anxious) were more prone to developing maladaptive coping schema, whereas the securely attached patients were more likely to recover without as many maladaptive coping skills.

Keywords: First episode psychosis; Trauma; Attachment; Anxious

\section{Erin K Idehenre ${ }^{1}$, Tracy Carpenter-Aeby ${ }^{1}$ and Victor G Aeby ${ }^{2 *}$}

1 School of Social Work, College of Health and Human Performance, East Carolina University, NC 27858-4353, USA

2 Department of Health Education and Promotion, College of Health and Human Performance, East Carolina University, NC 27858, USA

\section{*Corresponding author: Victor G Aeby \\ - aebyv@ecu.edu}

Department of Health Education and Promotion, College of Health and Human Performance, East Carolina University, 2205 Carol Belk Building, Greenville, NC 27858, USA.

Tel: (252) 328-4650

Citation: Idehenre EK, Carpenter-Aeby T, Aeby VG (2017) The Effect of Attachment on Post-psychotic Trauma in First Episode Psychosis. Acta Psychopathol. Vol. 3 No. 4:43

Received: June 15, 2017; Accepted: July 12, 2017; Published: July 20, 2017

\section{Introduction}

The goal of this literature review is to discover how attachment affects any trauma that is associated with first episode psychosis. Learning how attachment affects post-psychotic trauma can assist therapists in treatment plans for these individuals to attain better outcomes in the therapeutic setting. Discovering the different types of trauma that are associated with psychosis, as well as any traumas that may predict a psychotic episode. Additionally, this review may help in finding a more comprehensive method of care for post-psychosis.

Bowlby's Theory of Attachment posits that early relationships have a major impact on interpersonal relationships in life [1]. The theory is that these early relationships form the internal working model of a person and that is the lens through which they view the world. This internal working model affects interpersonal relationships as well as social functioning of the person through their lifetime. Each study selected was chosen from keywords in the title, specifically because the role that attachment plays in the outcome of post-psychotic trauma is the research we wanted to discover. The theoretical implications are that when in individual or group therapy, clients that have had a psychotic episode may need to be guided through attachment work as well as processing their psychotic episode and the trauma that resulted from the episode. By working on attachment, the client may experience less post-psychotic shame as well as fewer negative symptoms of psychosis such as apathy, social withdrawal, or anhedonia. In short, working not only on the trauma but also on attachment, the client may be able to experience a fuller life. The results of this study may impact social work practice and policy, as current standards of care are evolving to trauma informed care as well as treatment of trauma such as trauma-focused cognitive behavioral theory, however attachment style may also be key in 
identifying roadblocks to recovery. Roadblocks to recovery are often maladaptive coping mechanisms and through the literature appear to be associated with insecurely attached clients, whether they are insecure avoidant/ambivalent or insecure anxious [2].

Attachment is the main theoretical model that was examined, and each of the articles utilized similar testing hypothesis, exploring how attachment style impacted the patient's recovery post-psychosis. Attachment is thought by many to be key in discovering how people recover from trauma. Psychotic trauma is difficult to define through the existing literature, however because psychosis is (for the patient) impossible to explain what is happening and why and therefore can be considered traumatic for many people. However, defining any psychotic break as automatically traumatic is also not necessarily true, as all clients are different in their response to traumatic events.

The types of childhood attachment are: secure, avoidant, and insecure. Within avoidant and insecure there are two subsets of additional attachment models. As a child grows into an adult these basic models of attachment are how the world is viewed, and how someone may react to their stresses. Bowlby and Ainsworth have done significant work in the field of attachment; however their focus was on the attachment of children [3]. Research then developed the idea of adult attachment, and this literature review seeks to understand a link between attachment and post-psychotic trauma. Because the research is based on how a person may view their world and react to their stressors, attachment may be an important factor in the recovery of a psychotic state. Psychosis is a major stressor to the individual as well as their family. Recovering from that stress will depend on what kind of attachment the individual has to each part of the family unit. Attachment may matter in how people can resume their lives, post-psychosis. However, as with most therapy and theories, perhaps it will be individual specific.

\section{Methods}

A systematic review of the literature was conducted to examine the relation of attachment to post-psychotic trauma. OneSearch database from East Carolina University was utilized to search for articles reviewed. OneSearch is a comprehensive database of all available resources through East Carolina University. The keywords for this search were, "trauma," "attachment," and "first episode psychosis." The authors limited articles to peer/ scholarly reviewed during the last 10 years (January 1, 2007 to March 1, 2017). Then the authors conduscted a face validity review by examining the titles and abstracts of the remaining articles to assure that they were germane to the three key words.

\section{Results}

As shown in Appendix 1, the research question for this literature review was "what is the effect of attachment style on postpsychotic trauma" with the anticipation of understanding better if and how attachment effects psychosis, and what modalities are preferred for treating patients with trauma related to their first episode of psychosis. As shown in Appendix 2, the results appeared consistent with the hypothesis that attachment does appear to matter in treating psychosis, especially as several of the studies showed that insecure attachment style can have a poor prognosis for a healthy recovery. The majority of these studies were clear and straightforward. Many of them had carefully considered conclusions with clear limitations and strengths and themes as shown in Appendix 3. Many of the studies that were in the "other" category appeared that they touched upon attachment and psychosis, but some missed the mark of having any clear conclusion.

There were 17,642 results for the three keywords. Limiting by "peer reviewed/ scholarly articles) the search rendered 1311 articles. The next limiter was time, so the search was changed to include January 1, 2007 to March 1, 2017. This search resulted in 738 articles. Then two authors conducted a face validity review by examining the title and abstract of each article to ensure fit into the literature review. Twenty-eight articles met all criteria. The qualifying studies are listed in Appendix 1, which include the themes: early trauma, attachment style, social anxiety disorder, mentalization, and a group of other articles that were singular in theme. These themes emerged following a thorough review of the articles that were selected. The theme with the most number of articles were about attachment $(n=12)$. The second most prevalent theme included articles written on early trauma $(n=6)$. Following that, the most prevalent theme concerned mentalization $(n=4)$. Social Anxiety Disorder was the fourth most prevalent theme $(n=2)$. The "other" category, "outliners," had one article each on mindfulness, symptomatology, metacognition, recovery, coping, and personality disorder. The research questions for this study, how many articles relate to attachment and does effect does attachment have on postpsychotic trauma seemed to be resolved in that the 28 resulting articles attachment style appear to shape the way a person sees the world, so to experience something as life-changing as psychosis, attachment likely changes the way the person views it. The authors eliminated articles from the 709 articles did not fit the search parameters, based on their title and abstract. Because of the specificity of the question, there was not as much current research on the topic. In fact, the studies that are in the "other" category are not necessarily written about attachment, but may have some information about the link between attachment and post-psychotic trauma. The evidence appears to support links between the way a person copes with psychosis and attachment style. While insecurely attached patients may show higher prevalence of paranoia, it is not indicative of every person. The experience of psychosis is highly personal and while attachment may play a role, it is not to be considered that it is the only factor in the recovery of psychosis.

Many of the studies were written by similar groups of people with similar research aims, and several of the studies cited each other, which may indicate homogeneity within the sample. However, with these limitations, the link between attachment and recovery from psychosis can likely be inferred. These studies carefully consider the link between the two ideas and many come to the conclusion that it is likely there is a relationship.

Appendix 1 shows each study in alphabetical order with how many participants of the study in the " $\mathrm{N}$ " column, the assessments 
or tests used to get results, and study outcomes. Many of the studies used similar assessments with some of the common ones being the Positive and Negative Syndrome Scale (PANSS), Adult Attachment Interview (AAI), and Psychosis Attachment Measure (PAMS). These instruments have been shown to be reliable for the purposes for which they were developed. Some of the studies utilized self-report, or created their own assessment tool. While not uncommon, most of those studies also listed their assessment tool or self-report as a limitation. One study listed the Adult Attachment Interview (AAI), as a limitation due to the invasive nature of the test itself. Appendix $\mathbf{3}$ discusses each study within its theme. The major themes of this literature review were: attachment style, early trauma, mentalization, and "other." In the "other" category were outliers that did not have much to do with the other themes. Additionally, some of these outliers had titles and abstracts that did not indicate the actual purpose of the study so they were included with the literature review [4].

Some articles $(n=12)$ discussed attachment style and psychotic trauma in this sample [5]. Each of the articles emphasized that attachment style does have an impact on how patients recover from psychosis. While mindfulness was not the next highest category, it is important to mention second, because it could provide a difference in the delivery of therapeutic modality. By using mindfulness, a practitioner may be able to assess the patient more quickly, and by providing a corrective emotional experience the patient may learn that it is possible to trust. All of the research is interwoven in that each piece affects another part of the research. Therefore, utilizing mentalization in combination with other therapy modalities may result in a more effective recovery for a patient no matter their attachment style. It is important to note that patients that are insecurely attached, whether it is avoidant or anxious appear that they are more likely to have a difficult recovery from psychosis. Securely attached patients appear to use that secure attachment as a resiliency factor in their recovery. Also of note is that each study mentioned that ultimately, it depends on the person that is being treated. Attachment style may make some symptoms more prevalent, but that it is not safe to assume that it is totally true. However, the studies clearly support that it is important to discuss attachment in the therapeutic setting and provide a corrective emotional experience as that may help with trust for the patient.

\section{References}

1 Bowlby J (1973) Separation: anxiety \& anger. Attachment and Loss ( $2^{\text {nd }}$ edn.). International psycho-analytical library no. 95, London: Hogarth Press.

2 Korver-Nieberg N, Berry K, Meijer CJ, Haan L (2014) Adult attachment and psychotic phenomenology in clinical and non-clinical samples: A systematic review. Psychol Psychother Theor Res Pract 87: 127-154

3 Bretherton I (1992) The origins of attachment theory: john bowlby and mary ainsworth. Dev Psychol 28: 759-775.

4 Berry K, Barrowclough C, Wearden A (2009) Adult attachment, perceived earlier experiences of care giving and trauma in people with psychosis. J Ment Health 18: 280-287.

\section{Discussion and Conclusion}

\section{Summary of findings}

The evidence presented appears to be overwhelming that the trauma associated with psychosis must be discussed within the therapeutic setting. The trauma associated with hospitalization is not to be discounted $[5,6]$ and should also be discussed in the therapeutic setting. Additionally, a client's attachment style will affect the therapeutic alliance [7]. Moreover, there are relatively few studies that examine attachment style in post-psychotic trauma, although the few studies that have been completed are evidence that more examination should be done.

\section{Limitations}

Limitations to this study are the small number of studies $(n=30)$. Further, there are few studies that examine attachment's role in post-psychotic trauma with first episode psychosis $(n=12)$ [810]. Ultimately, this is an area that needs further research due to the clear connection between attachment type and recovery, particularly when a patient has insecure avoidant or anxious attachment. Additionally, many of the limitations of the studies utilized stated that while attachment might matter, ultimately it will depend on the individual and more factors than can be controlled in one study.

\section{Application to social work policy and practice}

Considering the professional training and skill versatility of social workers, they may be in unique positions to successfully work with patients coping with post-psychotic trauma $[11,12]$. Social Workers have the ability to create correctional relational experiences and utilize mentalization to foster a therapeutic alliance so that post-psychotic trauma can be processed and reduced. Utilizing mentalization and fostering that skill with the patient may assist patients in recovery [7]. Another important application to Social Work practice is that trauma informed care is necessary, particularly in the severely and persistently mentally ill population, as many of these articles underscore how past trauma affects recovery post-psychosis. Mentalization-based therapy may be an important type of therapy to include when treating those with a psychotic spectrum disorder, because it has the potential to aid the patient in reality based thought.

5 Gumley Al, Taylor HEF, Schwannauer M, MacBeth A (2014) A systematic review of attachment and psychosis: measurement, construct validity and outcomes. Acta Psychiatr Scand 129: 257-274.

6 Berry K, Ford S, Jellicoe-Jones L, Haddock G (2015) Trauma in relation to psychosis and hospital experiences: the role of past trauma and attachment. Psychol Psychother Theor Res Pract 88: 227-239

7 Debbané M, Benmiloud J, Salaminios G, Solida-Tozzi A, Armando M, et al. (2016) Mentalization-based treatment in clinical high-risk for psychosis: a rationale and clinical illustration. J Contemp Psychother 46: 217-225.

8 Lambert M, Conus P, Lambert T, McGorry PD (2003) Pharmacotherapy of first-episode psychosis. Expert Opin Pharmacother 4: 717-50. 
9 Franz L, Carter T, Leiner AS, Bergner E, Nancy J, et al. (2010) Stigma and treatment delay in first-episode psychosis: a grounded theory study. Early Interv Psychiatry 4: 47-56.

10 Kane JM, Schooler NR, Marcy P, Correll CU, Brunette MF, et al. (2015) The RAISE early treatment program for first-episode psychosis: background, rationale, and study design. J Clin Psychiatry 76: 240-246.
11 Arango C (2015) First-episode psychosis research: time to move forward (by Looking Backwards). Schizophr Bull 41: 1205-1206.

12 McgorrY PD, Killackey E, Yung A (2008) Early intervention in psychosis: concepts, evidence and future directions. World Psychiatry 7: 148-156. 
Appendix 1 Matrix for systematic literature review $(n=28)$.

\begin{tabular}{|c|c|c|c|c|c|}
\hline & Study & $\mathbf{N}$ & $\begin{array}{c}\text { Assessment } \\
\text { Tool }\end{array}$ & Type of Study & Outcomes \\
\hline 1 & $\begin{array}{c}\text { Aas, M., Henry, C., Bellivier, F., } \\
\text { Lajnef, M., Gard, S., Kahn, J., } \\
\text { Etain, B. (2016). Affective } \\
\text { lability mediates the } \\
\text { association between childhood } \\
\text { trauma and suicide attempts, } \\
\text { mixed episodes and co-morbid } \\
\text { anxiety disorders in bipolar } \\
\text { disorders. Psychological } \\
\text { Medicine, } 47(5), 902-11 . \\
\text { doi:10.1017/S00332917160030 } \\
81 .\end{array}$ & 342 & $\begin{array}{c}\text { Diagnostic } \\
\text { Interview for } \\
\text { Genetic } \\
\text { Studies } \\
\text { (DIGS) } \\
\text { Affective } \\
\text { Lability Scale } \\
\text { (ALS-SF) } \\
\text { Childhood } \\
\text { Trauma } \\
\text { Questionnair } \\
\text { e (CTQ) }\end{array}$ & Quantitative & $\begin{array}{l}\text { Childhood trauma } \\
\text { influences affect } \\
\text { lability as well as } \\
\text { number of suicide } \\
\text { attempts }\end{array}$ \\
\hline 2 & $\begin{array}{c}\text { Bentall, R. P., \& Fernyhough, C. } \\
\text { (2008). Social predictors of } \\
\text { psychotic experiences: } \\
\text { Specificity and psychological } \\
\text { mechanisms. Schizophrenia } \\
\text { Bulletin, 34(6), 1012-1020. } \\
\text { doi:10.1093/schbul/sbn103. }\end{array}$ & $\mathrm{n} / \mathrm{a}$ & $n / a$ & $\begin{array}{c}\text { Summary of } \\
\text { thoughts }\end{array}$ & $\begin{array}{l}\text { When treating } \\
\text { mental illness, it } \\
\text { is important to } \\
\text { remember the } \\
\text { connections } \\
\text { between } \\
\text { biological and } \\
\text { environmental } \\
\text { issues. }\end{array}$ \\
\hline 3 & $\begin{array}{c}\text { Berry, K., Barrowclough, C., \& } \\
\text { Wearden, A. (2008). } \\
\text { Attachment theory: A } \\
\text { framework for understanding } \\
\text { symptoms and interpersonal } \\
\text { relationships in psychosis. } \\
\text { Behaviour Research and } \\
\text { Therapy, } 46(12), 1275-1282 .\end{array}$ & 96 & $\begin{array}{l}\text { Psychosis } \\
\text { Attachment } \\
\text { Measure } \\
\text { (PAM) } \\
\text { Positive and } \\
\text { Negative } \\
\text { Syndrome } \\
\text { Scale (PANSS) }\end{array}$ & Quantitative & $\begin{array}{l}\text { Associations } \\
\text { between positive } \\
\text { symptoms of } \\
\text { psychosis and } \\
\text { avoidant } \\
\text { attachment, } \\
\text { associations } \\
\text { between }\end{array}$ \\
\hline
\end{tabular}




\begin{tabular}{|c|c|c|c|c|c|}
\hline & doi:10.1016/j.brat.2008.08.009 & & $\begin{array}{l}\text { Inventory of } \\
\text { Personal } \\
\text { Problems } \\
\text { (IPP) } \\
\text { Social } \\
\text { Behavior } \\
\text { Scale (SBS) } \\
\text { Working } \\
\text { Alliance } \\
\text { Inventory } \\
\text { (WAI) }\end{array}$ & & $\begin{array}{c}\text { avoidant and } \\
\text { dismissive } \\
\text { attachment styles } \\
\text { and lack of } \\
\text { therapeutic } \\
\text { alliance. }\end{array}$ \\
\hline 4 & $\begin{array}{l}\text { Berry, K., Barrowclough, C., \& } \\
\text { Wearden, A. (2009). Adult } \\
\text { attachment, perceived earlier } \\
\text { experiences of care giving and } \\
\text { trauma in people with } \\
\text { psychosis. Journal of Mental } \\
\text { Health, 18(4), 280-287. } \\
\text { doi:10.1080/09638230701879 } \\
185 .\end{array}$ & 80 & $\begin{array}{c}\text { PAM, } \\
\text { Parental } \\
\text { Bonding } \\
\text { Instrument } \\
\text { (PBI), Trauma } \\
\text { History } \\
\text { Questionnair } \\
\text { e (THQ), } \\
\text { PANSS, }\end{array}$ & Qualitative & $\begin{array}{l}\text { Correlation } \\
\text { between trauma } \\
\text { and anxious } \\
\text { attachment, did } \\
\text { not find } \\
\text { correlation } \\
\text { between } \\
\text { avoidant } \\
\text { attachment and } \\
\text { early } \\
\text { interpersonal } \\
\text { traumas }\end{array}$ \\
\hline 5 & $\begin{array}{l}\text { Berry, K., Ford, S., Jellicoe-Jones, L., } \\
\text { \& Haddock, G. (2015). Trauma } \\
\text { in relation to psychosis and } \\
\text { hospital experiences: The role } \\
\text { of past trauma and } \\
\text { attachment. Psychology and } \\
\text { Psychotherapy: Theory, } \\
\text { Research and Practice, } 88(3), \\
227-239 . \\
\text { doi:10.1111/papt.12035. }\end{array}$ & 50 & $\begin{array}{l}\text { PANSS, THQ, } \\
\text { The Impact of } \\
\text { Event Scale- } \\
\text { Revised (IES- } \\
\text { R), } \\
\text { Psychiatric } \\
\text { Experiences } \\
\text { Questionnair } \\
\text { e (PEQ), PAM }\end{array}$ & $\begin{array}{l}\text { Quantitative, } \\
\text { multiple } \\
\text { regression } \\
\text { analysis }\end{array}$ & $\begin{array}{l}\text { PTSD related to } \\
\text { psychosis, as well } \\
\text { as PTSD related } \\
\text { to hospitalization: } \\
\text { significant } \\
\text { correlation } \\
\text { between } \\
\text { attachment } \\
\text { anxiety and PTSD } \\
\text { symptoms from } \\
\text { both psychosis } \\
\text { and }\end{array}$ \\
\hline
\end{tabular}




\begin{tabular}{|c|c|c|c|c|c|}
\hline & & & & & $\begin{array}{l}\text { hospitalization. } \\
\text { Suggests a need } \\
\text { to discuss both } \\
\text { trauma from } \\
\text { psychosis and } \\
\text { from } \\
\text { hospitalization } \\
\text { with patient. }\end{array}$ \\
\hline 6 & $\begin{array}{l}\text { Brent, B. K. (2015). A Mentalization- } \\
\text { Based approach to the } \\
\text { development of the } \\
\text { therapeutic alliance in the } \\
\text { treatment of schizophrenia. } \\
\text { Journal of Clinical Psychology, } \\
71(2), 146-156 . \\
\text { doi:10.1002/jclp.22150. }\end{array}$ & $\mathrm{n} / \mathrm{a}$ & $\mathrm{N} / \mathrm{A}$ & Case Study & $\begin{array}{l}\text { Mentalization } \\
\text { approach to } \\
\text { treating acute } \\
\text { schizophrenia } \\
\text { with short term } \\
\text { benefits, but long } \\
\text { term outcome is } \\
\text { "guarded" }\end{array}$ \\
\hline 7 & $\begin{array}{c}\text { Bucci, S., Emsley, R., \& Berry, K. } \\
\text { (2017). Attachment in } \\
\text { psychosis: A latent profile } \\
\text { analysis of attachment styles } \\
\text { and association with symptoms } \\
\text { in a large psychosis cohort. } \\
\text { Psychiatry Research, 247, 243- } \\
249 . \\
\text { doi:10.1016/j.psychres.2016.1 } \\
1.036 .\end{array}$ & 588 & $\begin{array}{c}\text { PAM, } \\
\text { PSYRATS, } \\
\text { THQ }\end{array}$ & Quantitative & $\begin{array}{l}\text { Attachment } \\
\text { patterns } \\
\text { mimicked healthy } \\
\text { population } \\
\text { numbers, each } \\
\text { attachment } \\
\text { pattern had a } \\
\text { calculable set of } \\
\text { differences. } \\
\text { Secure } \\
\text { Attachment: less } \\
\text { symptomatic } \\
\text { overall, lower } \\
\text { delusions, lower } \\
\text { hallucinations, } \\
\text { lowest reported } \\
\text { physical/sexual } \\
\text { abuse. }\end{array}$ \\
\hline
\end{tabular}




\begin{tabular}{|c|c|c|c|c|c|}
\hline 8 & $\begin{array}{l}\text { Burke, E., Danquah, A., \& Berry, K. } \\
\text { (2015). A qualitative } \\
\text { exploration of the use of } \\
\text { attachment theory in adult } \\
\text { psychological therapy. Clinical } \\
\text { Psychology \& Psychotherapy, } \\
\text { 23(2), 142-154. } \\
\text { doi:10.1002/cpp.1943. }\end{array}$ & 12 & $\begin{array}{l}\text { Therapists } \\
\text { that had an } \\
\text { interest in } \\
\text { attachment } \\
\text { theory were } \\
\text { sent an } \\
\text { interview } \\
\text { with specific } \\
\text { questions }\end{array}$ & Qualitative & $\begin{array}{l}\text { Attachment } \\
\text { theory can be } \\
\text { helpful in } \\
\text { developing the } \\
\text { therapeutic } \\
\text { alliance and } \\
\text { bettering client } \\
\text { outcomes. }\end{array}$ \\
\hline 9 & $\begin{array}{l}\text { Debbané, M., Benmiloud, J., } \\
\text { Salaminios, G., Solida-Tozzi, A., } \\
\text { Armando, M., Fonagy, P., \& } \\
\text { Bateman, A. (2016). } \\
\text { Mentalization-based treatment } \\
\text { in clinical high-risk for } \\
\text { psychosis: A rationale and } \\
\text { clinical illustration. Journal of } \\
\text { Contemporary Psychotherapy, } \\
46(4), 217-225 . \\
\text { doi:10.1007/s10879-016-9337- } \\
4 .\end{array}$ & $\mathrm{n} / \mathrm{a}$ & $\mathrm{n} / \mathrm{a}$ & Case Study & $\begin{array}{l}\text { Utilizing } \\
\text { Mentalization } \\
\text { Based Treatment } \\
\text { (MBT) for } \\
\text { Cognitive High- } \\
\text { Risk patients } \\
\text { (CHR) offer them } \\
\text { the chance to } \\
\text { reframe their } \\
\text { thinking to a "less } \\
\text { rigid, delusional, } \\
\text { and perseverative } \\
\text { patterns of reality } \\
\text { testing." }\end{array}$ \\
\hline 10 & $\begin{array}{c}\text { Fett, A. J., Shergill, S. S., Korver- } \\
\text { Nieberg, N., Yakub, F., } \\
\text { Gromann, P. M., \& } \\
\text { Krabbendam, L. (2016). } \\
\text { Learning to trust: Trust and } \\
\text { attachment in early psychosis. } \\
\text { Psychological Medicine, 46(7), } \\
\text { 1437-11. } \\
\text { doi:10.1017/S00332917160000 }\end{array}$ & $\begin{array}{c}\text { 39:100 } \\
\text { Early } \\
\text { psychosis: } \\
\text { healthy }\end{array}$ & $\begin{array}{c}\text { PAM, "The } \\
\text { Trust Game", } \\
\text { PANSS, Green } \\
\text { Paranoid } \\
\text { Thoughts } \\
\text { Scale (GPTS) }\end{array}$ & Quantitative & $\begin{array}{l}\text { Those with early } \\
\text { psychosis } \\
\text { displayed less } \\
\text { trust than their } \\
\text { healthy } \\
\text { counterparts, } \\
\text { however were } \\
\text { able to adapt to } \\
\text { their healthy } \\
\text { counterparts } \\
\text { "positive social } \\
\text { cues" }\end{array}$ \\
\hline
\end{tabular}




\begin{tabular}{|c|c|c|c|c|c|}
\hline 11 & $\begin{array}{l}\text { Gumley, A. I., Schwannauer, M., } \\
\text { Macbeth, A., Fisher, R., Clark, } \\
\text { S., Rattrie, L., Birchwood, M. } \\
\text { (2014). Insight, duration of } \\
\text { untreated psychosis and } \\
\text { attachment in first-episode } \\
\text { psychosis: Prospective study of } \\
\text { psychiatric recovery over 12- } \\
\text { month follow-up. The British } \\
\text { Journal of Psychiatry: The } \\
\text { Journal of Mental Science, } \\
\text { 205(1), 60-67. } \\
\text { doi:10.1192/bjp.bp.113.12672 } \\
2 .\end{array}$ & 54 & $\begin{array}{c}\text { Adult } \\
\text { Attachment } \\
\text { Interview } \\
\text { (AAI), PANSS }\end{array}$ & Quantitative & $\begin{array}{l}\text { Attachment style } \\
\text { affects recovery } \\
\text { of first episode } \\
\text { psychosis. }\end{array}$ \\
\hline 12 & $\begin{array}{l}\text { Gumley, A. I., Taylor, H. E. F., } \\
\text { Schwannauer, M., \& MacBeth, } \\
\text { A. (2014). A systematic review } \\
\text { of attachment and psychosis: } \\
\text { Measurement, construct } \\
\text { validity and outcomes. Acta } \\
\text { Psychiatrica Scandinavica, } \\
\text { 129(4), 257-274. } \\
\text { doi:10.1111/acps.12172. }\end{array}$ & $n / a$ & $\begin{array}{l}\text { Library } \\
\text { search }\end{array}$ & Case Study & $\begin{array}{l}\text { First episode of } \\
\text { psychosis } \\
\text { damages the } \\
\text { attachment } \\
\text { ability of the } \\
\text { client, due to the } \\
\text { lack of ability to } \\
\text { mentalize. }\end{array}$ \\
\hline 13 & $\begin{array}{l}\text { Huguelet, P., Mohr, S., Rieben, I., } \\
\text { Hasler, R., Perroud, N., \& } \\
\text { Brandt, P. (2015). Attachment } \\
\text { and coping in psychosis in } \\
\text { relation to spiritual figures. } \\
\text { BMC Psychiatry, 15(1), } 237 . \\
\text { doi:10.1186/s12888-015-0617- } \\
4 .\end{array}$ & $\begin{array}{l}48 \text { ( } 30 \text { with } \\
\text { psychosis, } \\
18 \text { healthy } \\
\text { controls) }\end{array}$ & AAI & Quantitative & $\begin{array}{l}\text { A majority of the } \\
\text { patients and } \\
\text { controls believed } \\
\text { in a spiritual } \\
\text { figure that } \\
\text { functioned as a } \\
\text { secure } \\
\text { attachment } \\
\text { figure, which in } \\
\text { patients helped } \\
\text { their positive }\end{array}$ \\
\hline
\end{tabular}




\begin{tabular}{|c|c|c|c|c|c|}
\hline & & & & & coping skills \\
\hline 14 & $\begin{array}{c}\text { Korver-Nieberg, N., Berry, K., Meijer, } \\
\text { C. J., \& Haan, L. (2014). Adult } \\
\text { attachment and psychotic } \\
\text { phenomenology in clinical and } \\
\text { non-clinical samples: A } \\
\text { systematic review. Psychology } \\
\text { and Psychotherapy: Theory, } \\
\text { Research and Practice, 87(2), } \\
\text { 127-154. } \\
\text { doi:10.1111/papt.12010. }\end{array}$ & $n / a$ & $\begin{array}{l}\text { Journal } \\
\text { search }\end{array}$ & $\begin{array}{l}\text { Literature } \\
\text { review }\end{array}$ & $\begin{array}{l}\text { Insecurely } \\
\text { attached (both } \\
\text { avoidant and } \\
\text { anxious) were } \\
\text { associated with } \\
\text { maladaptive } \\
\text { coping strategies }\end{array}$ \\
\hline 15 & $\begin{array}{l}\text { Lysaker, P. H., Bob, P., Pec, O., } \\
\text { Hamm, J., Kukula, M., Vohs, J., } \\
\text { Dimaggio, G. (2013). Synthetic } \\
\text { metacognition as a link } \\
\text { between brain and behavior in } \\
\text { schizophrenia. Translational } \\
\text { Neuroscience, 4(3), 368-377. } \\
\text { doi:10.2478/s13380-013-0131- } \\
4 .\end{array}$ & $n / a$ & $\begin{array}{l}\text { Journal } \\
\text { Search }\end{array}$ & $\begin{array}{l}\text { Literature } \\
\text { Review }\end{array}$ & $\begin{array}{l}\text { Examining } \\
\text { metacognition } \\
\text { ability in patients } \\
\text { with } \\
\text { schizophrenia } \\
\text { and encouraging } \\
\text { practitioners to } \\
\text { develop this skill. }\end{array}$ \\
\hline 16 & $\begin{array}{c}\text { Mathews, S., Onwumere, J., Bissoli, } \\
\text { S., Ruggeri, M., Kuipers, E., \& } \\
\text { Valmaggia, L. (2014). } \\
\text { Measuring attachment and } \\
\text { parental bonding in psychosis } \\
\text { and its clinical implications. } \\
\text { Epidemiology and Psychiatric } \\
\text { Sciences, , 1-8. } \\
\text { doi:10.1017/S20457960140007 } \\
30 .\end{array}$ & $n / a$ & $\begin{array}{l}\text { Self-Report } \\
\text { Questionnair } \\
\text { es and semi- } \\
\text { structured } \\
\text { interviews }\end{array}$ & Qualitative & $\begin{array}{l}\text { Indicate a } \\
\text { relationship } \\
\text { between } \\
\text { childhood } \\
\text { attachment } \\
\text { (especially early } \\
\text { memories) and } \\
\text { post-psychosis } \\
\text { outcomes }\end{array}$ \\
\hline 17 & $\begin{array}{c}\text { Michail, M., \& Birchwood, M. (2013). } \\
\text { Social anxiety disorder and } \\
\text { shame cognitions in psychosis. }\end{array}$ & 84 & $\begin{array}{c}\text { Social } \\
\text { Interaction } \\
\text { Anxiety Scale }\end{array}$ & $\begin{array}{l}\text { Quantitative, } \\
\text { comparing } \\
\text { socially }\end{array}$ & $\begin{array}{l}\text { Cognitive } \\
\text { Behavioral } \\
\text { Therapy is the }\end{array}$ \\
\hline
\end{tabular}




\begin{tabular}{|c|c|c|c|c|c|}
\hline & $\begin{array}{c}\text { Psychological Medicine, } 43(1) \text {, } \\
\text { 1-10. } \\
\text { doi:10.1017/S00332917120011 } \\
46 .\end{array}$ & & $\begin{array}{c}\text { (SIAS), Social } \\
\text { Phobia Scale } \\
\text { (SPS), PANSS, } \\
\text { Personal } \\
\text { Beliefs about } \\
\text { Illness } \\
\text { Questionnair } \\
\text { e (PBIQ), } \\
\text { Other as } \\
\text { Shamer Scale } \\
\text { (OAS), Social } \\
\text { Comparison } \\
\text { Scale (SCS), }\end{array}$ & $\begin{array}{l}\text { anxious } \\
\text { people with } \\
\text { psychosis and } \\
\text { without }\end{array}$ & $\begin{array}{l}\text { preferred } \\
\text { modality for } \\
\text { socially anxious } \\
\text { people, whether } \\
\text { they are } \\
\text { psychotic or not }\end{array}$ \\
\hline 18 & $\begin{array}{l}\text { Phillips, L. J., Francey, S. M., } \\
\text { Edwards, J., \& McMurray, N. } \\
\text { (2009). Strategies used by } \\
\text { psychotic individuals to cope } \\
\text { with life stress and symptoms } \\
\text { of illness: A systematic review. } \\
\text { Anxiety, Stress \& Coping, 22(4), } \\
\quad 371-410 . \\
\text { doi:10.1080/10615800902811 } \\
065 .\end{array}$ & 85 studies & $\mathrm{n} / \mathrm{a}$ & $\begin{array}{l}\text { Literature } \\
\text { Review }\end{array}$ & $\begin{array}{l}\text { This study } \\
\text { discusses that } \\
\text { most people } \\
\text { utilize coping } \\
\text { skills, but there is } \\
\text { not one that is } \\
\text { universally useful, } \\
\text { although task } \\
\text { oriented and } \\
\text { social avoidance } \\
\text { have more of a } \\
\text { positive impact } \\
\text { than emotion } \\
\text { oriented coping }\end{array}$ \\
\hline 19 & $\begin{array}{l}\text { Pos, K., Bartels-Velthuis, A. A., } \\
\text { Simons, C. J. P., Korver- } \\
\text { Nieberg, N., Meijer, C. J., de } \\
\text { Haan, L., (2015). Theory of } \\
\text { mind and attachment styles in } \\
\text { people with psychotic } \\
\text { disorders, their siblings, and } \\
\text { controls. Australian and New }\end{array}$ & $\begin{array}{l}111 \text { with } \\
\text { diagnosis } \\
\text { of } \\
\text { Schizophre } \\
\text { nia, } 106 \\
\text { non- } \\
\text { affected } \\
\text { siblings, } 63\end{array}$ & $\begin{array}{l}\text { PAM, } \\
\text { Conflicting } \\
\text { Beliefs and } \\
\text { Emotions, } \\
\text { Wesclser } \\
\text { Adult } \\
\text { Intelligence } \\
\text { Scale, CTQ }\end{array}$ & Quantitative & $\begin{array}{l}\text { The patients were } \\
\text { more avoidant or } \\
\text { anxiously } \\
\text { attached than } \\
\text { their healthy } \\
\text { siblings or } \\
\text { controls. These } \\
\text { patients also did }\end{array}$ \\
\hline
\end{tabular}




\begin{tabular}{|c|c|c|c|c|c|}
\hline & $\begin{array}{c}\text { Zealand Journal of Psychiatry, } \\
449(2), 171-180 . \\
\text { doi:10.1177/00048674145463 } \\
86 .\end{array}$ & controls & $\begin{array}{l}\text { (short form), } \\
\text { Community } \\
\text { assessment } \\
\text { of Psychic } \\
\text { Experiences } \\
\text { and PANSS }\end{array}$ & & $\begin{array}{l}\text { worse than their } \\
\text { siblings on } \\
\text { cognitive and } \\
\text { affective scales }\end{array}$ \\
\hline 20 & $\begin{array}{l}\text { Ringer, J. M., Buchanan, E. E., } \\
\text { Olesek, K., \& Lysaker, P. H. } \\
\text { (2014). Anxious and avoidant } \\
\text { attachment styles and } \\
\text { indicators of recovery in } \\
\text { schizophrenia: Associations } \\
\text { with self-esteem and hope. } \\
\text { Psychology and Psychotherapy: } \\
\text { Theory, Research and Practice, } \\
\quad 87(2), 209-221 . \\
\text { doi:10.1111/papt.12012. }\end{array}$ & $\begin{array}{c}52 \text { men } \\
\text { with } \\
\text { schizophre } \\
\text { nia, } 26 \text { with } \\
\text { HIV/AIDS }\end{array}$ & $\begin{array}{c}\text { ECR, } \\
\text { Rosenberg } \\
\text { Self-Estemm } \\
\text { Scale, Beck } \\
\text { Hopelessness } \\
\text { Scale, PANSS }\end{array}$ & Quantitative & $\begin{array}{l}\text { Attachment style } \\
\text { may impact some } \\
\text { parts of recovery } \\
\text { from psychosis }\end{array}$ \\
\hline 21 & $\begin{array}{l}\text { Smith, P. N., Gamble, S. A., Cort, N. } \\
\text { A., Ward, E. A., Conwell, Y., \& } \\
\text { Talbot, N. L. (2012). The } \\
\text { relationships of attachment } \\
\text { style and social maladjustment } \\
\text { to death ideation in depressed } \\
\text { women with a history of } \\
\text { childhood sexual abuse. } \\
\text { Journal of Clinical Psychology, } \\
68(1), 78-87 . \\
\text { doi:10.1002/jclp.20852. }\end{array}$ & 106 & $\begin{array}{c}\text { Experiences } \\
\text { in Close } \\
\text { Relationships } \\
\text { Scale (ECR), } \\
\text { Social } \\
\text { Adjustment } \\
\text { Scale (SAS), } \\
\text { Hamilton } \\
\text { Rating Scale } \\
\text { for } \\
\text { Depression } \\
\text { (HRSD) }\end{array}$ & Quantitative & $\begin{array}{l}\text { There are strong } \\
\text { associations } \\
\text { between "death } \\
\text { ideation" and } \\
\text { "social } \\
\text { maladjustment" } \\
\text { and some anxious } \\
\text { and avoidant } \\
\text { attachment styles } \\
\text { in women who } \\
\text { have BPD. }\end{array}$ \\
\hline 22 & $\begin{array}{l}\text { Stein, H., \& Allen, J. G. (2007). } \\
\text { Mentalizing as a framework for } \\
\text { integrating therapeutic } \\
\text { exposure and relationship } \\
\text { repair in the treatment of a }\end{array}$ & $\mathrm{n} / \mathrm{a}$ & $\begin{array}{l}\text { Discussion of } \\
\text { exposure } \\
\text { therapy vs } \\
\text { mentalization }\end{array}$ & Case study & $\begin{array}{l}\text { This article proves } \\
\text { nothing. }\end{array}$ \\
\hline
\end{tabular}




\begin{tabular}{|c|c|c|c|c|c|}
\hline & $\begin{array}{c}\text { patient with complex } \\
\text { posttraumatic } \\
\text { psychopathology. Bulletin of } \\
\text { the Menninger Clinic, } 71(4) \text {, } \\
273-290 \text {. } \\
\text { doi:10.1521/bumc.2007.71.4.2 } \\
73 .\end{array}$ & & & & \\
\hline 23 & $\begin{array}{c}\text { Tan, R., Gould, R. V., Combes, H., \& } \\
\text { Lehmann, S. (2014). Distress, } \\
\text { trauma, and recovery: } \\
\text { Adjustment to first episode } \\
\text { psychosis. Psychology and } \\
\text { Psychotherapy: Theory, } \\
\text { Research and Practice, 87(1), } \\
\text { 80-95. doi:10.1111/j.2044- } \\
\text { 8341.2012.02073.x }\end{array}$ & 8 & $\begin{array}{l}\text { Semi- } \\
\text { structured } \\
\text { interviews }\end{array}$ & Qualitative & \\
\hline 24 & $\begin{array}{l}\text { Tiliopoulos, N., \& Goodall, K. (2009). } \\
\text { The neglected link between } \\
\text { adult attachment and } \\
\text { schizotypal personality traits. } \\
\text { Personality and Individual } \\
\text { Differences, } 47(4), 299-304 . \\
\text { doi:10.1016/j.paid.2009.03.017 } \\
.\end{array}$ & 161 & $\begin{array}{l}\text { Schizotypal } \\
\text { Personality } \\
\text { Questionnair } \\
\text { e (SPQ), and } \\
\text { ECR }\end{array}$ & Quantitative & $\begin{array}{l}\text { Avoidant } \\
\text { attachment } \\
\text { seems to be } \\
\text { associated with } \\
\text { only negative } \\
\text { symptoms in } \\
\text { psychosis, while } \\
\text { anxious } \\
\text { attachment was } \\
\text { moderately } \\
\text { associated with } \\
\text { both positive and } \\
\text { negative } \\
\text { symptoms }\end{array}$ \\
\hline 25 & $\begin{array}{c}\text { Turner, K. (2009). Mindfulness: The } \\
\text { present moment in clinical } \\
\text { social work. Clinical Social } \\
\text { Work Journal, 37(2), 95-103. }\end{array}$ & $n / a$ & mindfulness & Case study & $\begin{array}{l}\text { The importance } \\
\text { of mindfulness in } \\
\text { clinical social } \\
\text { work, for the }\end{array}$ \\
\hline
\end{tabular}




\begin{tabular}{|c|c|c|c|c|c|}
\hline & $\begin{array}{c}\text { doi:10.1007/s10615-008-0182- } \\
0 .\end{array}$ & & & & social worker \\
\hline 26 & $\begin{array}{l}\text { van Dam, D. S., Korver-Nieberg, N., } \\
\text { Velthorst, E., Meijer, C. J., de } \\
\text { Haan, L., \& For Genetic Risk } \\
\text { and Outcome in Psychosis, } \\
\text { (GROUP). (2014). Childhood } \\
\text { maltreatment, adult } \\
\text { attachment and psychotic } \\
\text { symptomatology: A study in } \\
\text { patients, siblings and controls. } \\
\text { Social Psychiatry and } \\
\text { Psychiatric Epidemiology, } \\
\text { 49(11), 1759-1767. } \\
\text { doi:10.1007/s00127-014-0894- } \\
\text { 0. }\end{array}$ & $\begin{array}{c}131 \\
\text { patients, } \\
123 \\
\text { siblings, } 72 \\
\text { controls }\end{array}$ & CTQ, PAM & Quantitative & $\begin{array}{l}\text { Childhood } \\
\text { maltreatment } \\
\text { was a predictor } \\
\text { for the severity of } \\
\text { positive and } \\
\text { negative } \\
\text { symptoms in the } \\
\text { patient } \\
\text { population }\end{array}$ \\
\hline 27 & $\begin{array}{r}\text { Wan, M. W., \& Green, J. (2009). The } \\
\text { impact of maternal } \\
\text { psychopathology on child- } \\
\text { mother attachment. Archives } \\
\text { of Women's Mental Health, } \\
12(3), 123-134 . \\
\text { doi:10.1007/s00737-009-0066- } \\
5 .\end{array}$ & $n / a$ & AAI & meta-analysis & $\begin{array}{l}\text { Children who } \\
\text { have mothers } \\
\text { with mental } \\
\text { illness do not } \\
\text { necessarily } \\
\text { develop } \\
\text { attachment } \\
\text { issues }\end{array}$ \\
\hline 28 & $\begin{array}{l}\text { Wickham, S., Sitko, K., \& Bentall, R. } \\
\text { P. (2014). Insecure attachment } \\
\text { is associated with paranoia but } \\
\text { not hallucinations in psychotic } \\
\text { patients: The mediating role of } \\
\text { negative self-esteem. } \\
\text { Psychological Medicine, } 45(7), \\
1495-1507 . \\
\text { doi:10.1017/S00332917140026 }\end{array}$ & $\begin{array}{l}176 \text { people } \\
\text { with } \\
\text { schizophre } \\
\text { nia } \\
\text { spectrum } \\
\text { disorders, } \\
113 \\
\text { healthy } \\
\text { controls }\end{array}$ & $\begin{array}{l}\text { Persecution } \\
\text { and } \\
\text { Deservedness } \\
\text { Scale (PaDS), } \\
\text { PANSS, Multi- } \\
\text { dimensional } \\
\text { Locus of } \\
\text { Control Scale } \\
\text { (MLCS), Self- }\end{array}$ & Quantitative & $\begin{array}{l}\text { There were } \\
\text { strong } \\
\text { associations } \\
\text { between insecure } \\
\text { attachment and } \\
\text { patients with } \\
\text { negative self- } \\
\text { esteem and } \\
\text { paranoia. }\end{array}$ \\
\hline
\end{tabular}




\begin{tabular}{|c|c|}
\hline 33. & $\begin{array}{c}\text { esteem } \\
\text { Rating Scale } \\
\text { (SERS), and } \\
\text { RQ } \\
\text { (measures } \\
\text { attachment } \\
\text { style) }\end{array}$ \\
\hline
\end{tabular}

Appendix 2 Synthesis of keywords for systematic literature review (SLR).

\begin{tabular}{|c|c|c|c|}
\hline Years & Keywords & Limiters & $\begin{array}{c}\text { Number of } \\
\text { Articles }\end{array}$ \\
\hline $\begin{array}{c}\text { Beginning of records } \\
\text { (approximately1920s) to } \\
2017\end{array}$ & $\begin{array}{c}\text { Trauma } \\
\text { Attachment } \\
\text { First episode psychosis }\end{array}$ & none & $N=17,642$ \\
\hline $\begin{array}{l}\text { Beginning of records } \\
\text { (approximately1920s) to } \\
2017\end{array}$ & $\begin{array}{c}\text { Trauma } \\
\text { Attachment } \\
\text { First episode psychosis }\end{array}$ & Peer Reviewed/ scholarly & $n=1316$ \\
\hline 2007-2017 & $\begin{array}{c}\text { Trauma } \\
\text { Attachment } \\
\text { First episode psychosis }\end{array}$ & $\begin{array}{l}\text { Date } \quad \text { (past } 10 \\
\quad \text { years 2007-2017) }\end{array}$ & $n=738$ \\
\hline 2007-2017 & $\begin{array}{c}\text { Trauma } \\
\text { Attachment } \\
\text { First episode psychosis }\end{array}$ & $\begin{array}{c}\text { Face validity } \\
\text { attachment style affecting trauma of } \\
\text { first episode psychosis in title }\end{array}$ & $\mathrm{n}=28$ \\
\hline
\end{tabular}

Searches engines: OneSearch database from East Carolina University libraries.

Appendix 3 Synthesis and themes of key articles $(n=28)$.

\begin{tabular}{|c|c|c|c|c|c|}
\hline & Author/Date & Theme of article & Purpose & $\begin{array}{c}\text { General } \\
\text { Comments }\end{array}$ & Strengths \& Limitations \\
\hline 1 & Katherine Berry, & Attachment Style & Examined any & This is the only & Limitation: sample is not \\
\hline
\end{tabular}




\begin{tabular}{|c|c|c|c|c|c|}
\hline & $\begin{array}{l}\text { Christine Barrowclough, } \\
\text { Alison Wearden, } 2008\end{array}$ & & $\begin{array}{l}\text { association between } \\
\text { attachment and } \\
\text { symptoms of } \\
\text { psychosis. }\end{array}$ & $\begin{array}{l}\text { study in this } \\
\text { review to point } \\
\text { to avoidant } \\
\text { attachment } \\
\text { being associated } \\
\text { with greater } \\
\text { symptomatology }\end{array}$ & $\begin{array}{l}\text { representative of entire } \\
\text { population. Any results that } \\
\text { may have occurred are likely } \\
\text { dynamically dependent on } \\
\text { one another. }\end{array}$ \\
\hline 2 & $\begin{array}{l}\text { Katherine Berry, } \\
\text { Christine Barrowclough, } \\
\text { Alison Wearden, } 2009\end{array}$ & Attachment Style & $\begin{array}{l}\text { Testing if there is a } \\
\text { relationship } \\
\text { between attachment } \\
\text { and psychotic } \\
\text { recovery. }\end{array}$ & $\begin{array}{c}\text { There may be an } \\
\text { association } \\
\text { between } \\
\text { attachment and } \\
\text { psychotic } \\
\text { experience. }\end{array}$ & $\begin{array}{l}\text { Limitations: the associations } \\
\text { between variables may be } \\
\text { dynamic, without causality. }\end{array}$ \\
\hline 3 & $\begin{array}{c}\text { Bucci, Sandra, Richard } \\
\text { Emsley, Katherine } \\
\text { Berry, } 2016\end{array}$ & Attachment Style & $\begin{array}{l}\text { Examining the } \\
\text { difference in } \\
\text { attachment styles to } \\
\text { recovery }\end{array}$ & $\begin{array}{l}\text { The secure } \\
\text { attachment style } \\
\text { was the most } \\
\text { resilient, with } \\
\text { insecure or } \\
\text { disorganized } \\
\text { attachment } \\
\text { styles having } \\
\text { difficulty with } \\
\text { recovery. }\end{array}$ & $\begin{array}{l}\text { Limiting patients to one } \\
\text { attachment style, when } \\
\text { people can show } \\
\text { characteristics of multiple } \\
\text { styles. Additionally, the } \\
\text { specificity of the groupings } \\
\text { may have resulted in } \\
\text { limitation bias. }\end{array}$ \\
\hline 4 & $\begin{array}{c}\text { Eilish Burke, Adam } \\
\text { Danquah, Katherine } \\
\text { Berry, } 2015\end{array}$ & Attachment Style & $\begin{array}{l}\text { Examining } \\
\text { implications for } \\
\text { practice of } \\
\text { attachment and } \\
\text { therapeutic practice. }\end{array}$ & $\begin{array}{l}\text { Providing a } \\
\text { secure base and } \\
\text { corrective } \\
\text { emotional } \\
\text { experience is } \\
\text { important in } \\
\text { therapeutic } \\
\text { intervention. }\end{array}$ & $\begin{array}{l}\text { Most of the therapists in the } \\
\text { study identified as } \\
\text { psychodynamic/ } \\
\text { psychoanalytic. Small } \\
\text { sample. }\end{array}$ \\
\hline
\end{tabular}




\begin{tabular}{|c|c|c|c|c|c|}
\hline 5 & $\begin{array}{l}\text { A.K.J Fett, S.S Shergill, } \\
\text { N. Korver-Nieberg, F. } \\
\text { Yakub, P.M. Gromann, } \\
\text { L. Krabbendam, } 2016\end{array}$ & $\begin{array}{c}\text { Attachment style } \\
\text { and trust }\end{array}$ & $\begin{array}{l}\text { Attachment } \\
\text { insecurity may mean } \\
\text { distrust and social } \\
\text { dysfunction. }\end{array}$ & $\begin{array}{l}\text { Each group had } \\
\text { higher levels of } \\
\text { attachment } \\
\text { insecurity, but } \\
\text { not attachment } \\
\text { avoidance. Basic } \\
\text { trust was lower } \\
\text { in patients than } \\
\text { in controls. }\end{array}$ & $\begin{array}{l}\text { All diagnoses of the } \\
\text { psychotic spectrum were in } \\
\text { the sample, instead of a } \\
\text { homogenous sample. } \\
\text { Additionally, the sample was } \\
\text { small. However, this study } \\
\text { indicates that even with } \\
\text { insecure attachment, trust } \\
\text { can be gained. }\end{array}$ \\
\hline 7 & $\begin{array}{c}\text { A.I. Gumley, H.E.F } \\
\text { Taylor, M. } \\
\text { Schwannauer, A. } \\
\text { MacBeth, } 2013\end{array}$ & Attachment Style & $\begin{array}{l}\text { Literature review } \\
\text { that examined } \\
\text { attachment in } \\
\text { individuals with } \\
\text { psychosis. }\end{array}$ & $\begin{array}{l}\text { Insecure } \\
\text { attachment may } \\
\text { make recovery } \\
\text { more difficult for } \\
\text { post-psychosis. }\end{array}$ & $\begin{array}{c}\text { Strengths: systematic } \\
\text { research, exclusion of non- } \\
\text { clinical data. } \\
\text { Limitations: search } \\
\text { parameters (language, age } \\
\text { of studies), bias. }\end{array}$ \\
\hline 8 & $\begin{array}{l}\text { Philippe Huguelet, } \\
\text { Sylvia MOhr, Isabelle } \\
\text { Rieben, Roland Hasler, } \\
\text { Nader Perroud, Pierre- } \\
\text { Yves Brandt, } 2015\end{array}$ & Attachment Style & $\begin{array}{l}\text { Examines the coping } \\
\text { ability of patients } \\
\text { with psychosis and } \\
\text { attachment to } \\
\text { spiritual figures. }\end{array}$ & $\begin{array}{l}\text { Spirituality can } \\
\text { be used as a } \\
\text { positive } \\
\text { resource for } \\
\text { those with } \\
\text { chronic } \\
\text { psychosis. }\end{array}$ & $\begin{array}{l}\text { Utilizing measures that are } \\
\text { sensitive (AAI) is a strength, } \\
\text { and a weakness as it may } \\
\text { have caused some drop out } \\
\text { of participants. }\end{array}$ \\
\hline 9 & $\begin{array}{c}\text { Nikie Korver-Nieberg, } \\
\text { Katherine Berry, Carin J. } \\
\text { Meijer, Lieuwe de }\end{array}$ & Attachment Style & $\begin{array}{l}\text { Insecure anxious and } \\
\text { insecure avoidant } \\
\text { attachment styles }\end{array}$ & $\begin{array}{l}\text { Attachment is an } \\
\text { important part } \\
\text { of treatment of }\end{array}$ & $\begin{array}{l}\text { All of the studies were cross- } \\
\text { sectional, and despite what } \\
\text { results indicate, not } 100 \% \text { of }\end{array}$ \\
\hline
\end{tabular}




\begin{tabular}{|c|c|c|c|c|c|}
\hline & Haan, 2014 & & $\begin{array}{c}\text { seem to both be part } \\
\text { of the psychotic } \\
\text { phenomenology. }\end{array}$ & psychosis. & $\begin{array}{l}\text { people with psychosis have } \\
\text { an insecure attachment } \\
\text { type. All of the assessments } \\
\text { were self-report, which can } \\
\text { end up with bias. }\end{array}$ \\
\hline 10 & $\begin{array}{c}\text { Jamie M. Ringer, Erin E. } \\
\text { Buchanan, Kyle Olesek, } \\
\text { Paul H. Lysaker, } 2014\end{array}$ & Attachment Style & $\begin{array}{l}\text { Examining symptom } \\
\text { severity in patients } \\
\text { with schizophrenia } \\
\text { in conjunction with } \\
\text { attachment style. }\end{array}$ & $\begin{array}{l}\text { Study indicates } \\
\text { that participants } \\
\text { with } \\
\text { schizophrenia } \\
\text { had higher levels } \\
\text { of anxious } \\
\text { attachment. }\end{array}$ & $\begin{array}{l}\text { Limitation was homogenous } \\
\text { sample; adult attachment } \\
\text { interview may have been } \\
\text { more accurate assessment. }\end{array}$ \\
\hline 11 & $\begin{array}{c}\text { Niko Tiliopoulos, Karen } \\
\text { Goodall, } 2006\end{array}$ & Attachment Style & $\begin{array}{l}\text { Examined } \\
\text { relationship } \\
\text { between schizotypal } \\
\text { personality traits } \\
\text { and adult } \\
\text { attachment. }\end{array}$ & $\begin{array}{l}\text { Insecure } \\
\text { attachment } \\
\text { appears to be } \\
\text { more common } \\
\text { with schizotypy } \\
\text { personality } \\
\text { traits. }\end{array}$ & $\begin{array}{l}\text { Sample did not have enough } \\
\text { diversity and was comprised } \\
\text { of non-psychiatric people. } \\
\text { However, the study showed } \\
\text { a correlation between } \\
\text { attachment style and } \\
\text { interpersonal schizotypy. }\end{array}$ \\
\hline 12 & $\begin{array}{l}\text { S. Wickham, K. Sitko, } \\
\text { R.P. Bentall, } 2014\end{array}$ & Attachment Style & $\begin{array}{l}\text { Examined } \\
\text { relationship } \\
\text { between attachment } \\
\text { style, paranoid belief } \\
\text { and hallucinations in } \\
\text { comparison with } \\
\text { healthy controls. }\end{array}$ & $\begin{array}{l}\text { Insecure } \\
\text { attachment } \\
\text { seemed to } \\
\text { predict } \\
\text { paranoia. }\end{array}$ & $\begin{array}{l}\text { Insecure attachment (both } \\
\text { anxious and avoidant) were } \\
\text { highly likely to have } \\
\text { paranoid beliefs. Limitations: } \\
\text { diagnosis itself may harm } \\
\text { self-esteem. }\end{array}$ \\
\hline 13 & $\begin{array}{c}\text { M. Aas, C. Henry, F. } \\
\text { Bellivier, M. Lajnef, S. } \\
\text { Gard, J-P Kahn, T.V. } \\
\text { Lagerberg, S.R. Aminoff, } \\
\text { T. Bjella, M. Leboyer, } \\
\text { O.A. Andreassen, I. }\end{array}$ & Early Trauma & $\begin{array}{l}\text { A more labile affect } \\
\text { may indicate more } \\
\text { severe clinical } \\
\text { features of bipolar } \\
\text { disorder, in addition } \\
\text { to childhood trauma. }\end{array}$ & $\begin{array}{l}\text { Assessing for } \\
\text { childhood abuse } \\
\text { should be an } \\
\text { important part } \\
\text { of an initial } \\
\text { assessment for }\end{array}$ & $\begin{array}{l}\text { None of the variables } \\
\text { actually established } \\
\text { causality. Longitudinal study } \\
\text { should be conducted on this } \\
\text { thesis. Data on childhood } \\
\text { trauma was collected in }\end{array}$ \\
\hline
\end{tabular}




\begin{tabular}{|c|c|c|c|c|c|}
\hline & Melle, B. Etain, 2017 & & $\begin{array}{l}\text { These links may } \\
\text { indicate an increased } \\
\text { risk of suicide } \\
\text { attempts. }\end{array}$ & $\begin{array}{l}\text { people with } \\
\text { labile affect. }\end{array}$ & $\begin{array}{l}\text { retrospect, not when the } \\
\text { patient was psychotic. }\end{array}$ \\
\hline 14 & $\begin{array}{l}\text { Katherine Berry, Sarah } \\
\text { Ford, Lorna Jellicoe- } \\
\text { Jones, Gillian Haddock, } \\
2015\end{array}$ & Early Trauma & $\begin{array}{c}\text { Examines both } \\
\text { psychosis related } \\
\text { and hospital related } \\
\text { PTSD in patients } \\
\text { with a psychotic } \\
\text { spectrum disorder. }\end{array}$ & $\begin{array}{l}\text { The severity of } \\
\text { the psychosis is } \\
\text { associated with } \\
\text { PTSD symptoms. } \\
\text { Additionally, } \\
\text { PTSD symptoms } \\
\text { and “anxiety in } \\
\text { attachment } \\
\text { relationships" } \\
\text { are positively } \\
\text { correlated. }\end{array}$ & $\begin{array}{l}\text { Limitations: participants } \\
\text { were free to choose if they } \\
\text { wanted to participate, } \\
\text { meaning that the numbers } \\
\text { may be over or under } \\
\text { represented. Additionally, } \\
\text { hospital related trauma may } \\
\text { be underrepresented. The } \\
\text { measure used (CTQ) is } \\
\text { potentially traumatizing as } \\
\text { well. } \\
\text { Strengths: clearly identified } \\
\text { two kinds of trauma, both } \\
\text { the trauma of the psychotic } \\
\text { episode and the trauma of } \\
\text { inpatient hospitalization. }\end{array}$ \\
\hline 16 & $\begin{array}{l}\text { Philip N. Smith, } \\
\text { Stephanie A. Gamble, } \\
\text { Natalie A. Cort, Erin A. } \\
\text { Ward, Yeates Conwell, } \\
\text { Nancy L. Talbot, } 2011\end{array}$ & Early Trauma & $\begin{array}{l}\text { Examining the } \\
\text { attachment of } \\
\text { people with death } \\
\text { ideation, and what } \\
\text { their social } \\
\text { adaptations are. }\end{array}$ & $\begin{array}{l}\text { Those with both } \\
\text { social } \\
\text { maladaptation } \\
\text { and death } \\
\text { ideation tended } \\
\text { to be anxious or }\end{array}$ & $\begin{array}{l}\text { Limitations: the age } \\
\text { variation in the sample, no } \\
\text { comparison groups, and } \\
\text { relying on self-report. }\end{array}$ \\
\hline
\end{tabular}




\begin{tabular}{|c|c|c|c|c|c|}
\hline & & & & $\begin{array}{c}\text { avoidant } \\
\text { attachment } \\
\text { styles. }\end{array}$ & \\
\hline 17 & $\begin{array}{l}\text { D. S. van Dam, N. } \\
\text { Korver-Nieberg, E. } \\
\text { Velthorst, C. J. Meijer, } \\
\text { L. de Haan, } 2014\end{array}$ & Early Trauma & $\begin{array}{l}\text { Examining the } \\
\text { relation between } \\
\text { childhood } \\
\text { maltreatment and } \\
\text { development of } \\
\text { psychosis. }\end{array}$ & $\begin{array}{l}\text { This study } \\
\text { examines not } \\
\text { only childhood } \\
\text { maltreatment, } \\
\text { but also looks at } \\
\text { the genetic } \\
\text { component of } \\
\text { development of } \\
\text { psychosis. }\end{array}$ & $\begin{array}{l}\text { While attachment may play } \\
\text { a role in the increase of } \\
\text { positive symptoms of } \\
\text { psychosis, it is not the only } \\
\text { predictor. Examining other } \\
\text { variables is necessary. }\end{array}$ \\
\hline 18 & $\begin{array}{l}\text { Ming Wai Wan, } \\
\text { Jonathan Green }\end{array}$ & Early Trauma & $\begin{array}{l}\text { How a mother's own } \\
\text { psychopathology will } \\
\text { impact her child's } \\
\text { attachment style. }\end{array}$ & $\begin{array}{l}\text { This study } \\
\text { refutes most of } \\
\text { the cited } \\
\text { research in that } \\
\text { many children } \\
\text { with mother's } \\
\text { on the psychotic } \\
\text { spectrum do not } \\
\text { develop } \\
\text { attachment } \\
\text { related issues. }\end{array}$ & $\begin{array}{l}\text { The limitation is that } \\
\text { children who do not develop } \\
\text { attachment issues have } \\
\text { multiple protective factors, } \\
\text { so the outcome depends on } \\
\text { the case at hand. }\end{array}$ \\
\hline 19 & Benjamin K Brent, 2014 & Mentalization & $\begin{array}{l}\text { Developing the } \\
\text { therapeutic alliance } \\
\text { in the treatment of } \\
\text { schizophrenia } \\
\text { utilizing } \\
\text { mentalization based } \\
\text { therapy. }\end{array}$ & $\begin{array}{c}\text { Using } \\
\text { mentalization } \\
\text { may help } \\
\text { outcomes in } \\
\text { treating people } \\
\text { with psychotic } \\
\text { spectrum } \\
\text { disorders. }\end{array}$ & $\begin{array}{l}\text { Further research should be } \\
\text { done on mentalization based } \\
\text { therapy for the recovery of } \\
\text { people with schizophrenia. }\end{array}$ \\
\hline 20 & Martin Debbane, Jallal & Mentalization & Discusses the & Explains & Mentalization based therapy \\
\hline
\end{tabular}




\begin{tabular}{|c|c|c|c|c|c|}
\hline & $\begin{array}{l}\text { Benmiloud, George } \\
\text { Salaminios, Alessandra } \\
\text { Solida-Tozzi, Marco } \\
\text { Armando, Peter } \\
\text { Fonagy, Anthony } \\
\text { Bateman, } 2016\end{array}$ & & $\begin{array}{l}\text { difference between } \\
\text { metacognition and } \\
\text { mentalization, as } \\
\text { well as provides a } \\
\text { case study on } \\
\text { mentalization based } \\
\text { therapy. }\end{array}$ & $\begin{array}{l}\text { carefully } \\
\text { "pretend mode" } \\
\text { of how a person } \\
\text { on the psychotic } \\
\text { spectrum may } \\
\text { attempt } \\
\text { mentalization. }\end{array}$ & $\begin{array}{l}\text { may be helpful in treating } \\
\text { patients on the psychotic } \\
\text { spectrum, however should } \\
\text { not be used as the only } \\
\text { modality. }\end{array}$ \\
\hline 21 & $\begin{array}{c}\text { Karin Pos, Agna A } \\
\text { Bartels-Velthius, } \\
\text { Claudia JP Simons, Nikie } \\
\text { Korver-Nieberg, Carin J } \\
\text { Meijer, Lieuwe de } \\
\text { Haan, } 2015\end{array}$ & Mentalization & $\begin{array}{l}\text { Comparing patients } \\
\text { on the schizophrenia } \\
\text { spectrum versus } \\
\text { their non-affected } \\
\text { siblings and control } \\
\text { group. }\end{array}$ & $\begin{array}{l}\text { The study } \\
\text { supports the } \\
\text { conclusion that } \\
\text { those with } \\
\text { anxious } \\
\text { attachment may } \\
\text { not be able to } \\
\text { mentalize as } \\
\text { well as those } \\
\text { with secure } \\
\text { attachment. }\end{array}$ & $\begin{array}{l}\text { This study appears to show a } \\
\text { link between attachment } \\
\text { style and "theory of mind". } \\
\text { However, the study also } \\
\text { states that the results of said } \\
\text { study should be interpreted } \\
\text { cautiously. }\end{array}$ \\
\hline & $\begin{array}{c}\text { M. Michail, M } \\
\text { Birchwood, } 2012\end{array}$ & $\begin{array}{c}\text { Social Anxiety } \\
\text { Disorder }\end{array}$ & $\begin{array}{l}\text { Examining the role } \\
\text { of shame in social } \\
\text { anxiety and } \\
\text { psychosis. }\end{array}$ & $\begin{array}{c}\text { There appears to } \\
\text { be a connection } \\
\text { between } \\
\text { experiencing } \\
\text { shame when } \\
\text { someone has a } \\
\text { psychotic }\end{array}$ & $\begin{array}{l}\text { This study cautions about } \\
\text { implying causation to the } \\
\text { results, because correlation } \\
\text { does not always imply } \\
\text { causation. }\end{array}$ \\
\hline
\end{tabular}




\begin{tabular}{|c|c|c|c|c|c|}
\hline & & & & $\begin{array}{l}\text { episode and also } \\
\text { has social } \\
\text { anxiety. }\end{array}$ & \\
\hline 23 & $\begin{array}{l}\text { M. Michail, M. } \\
\text { Birchwood, } 2014\end{array}$ & $\begin{array}{c}\text { Social Anxiety } \\
\text { Disorder }\end{array}$ & $\begin{array}{l}\text { Examining the role } \\
\text { of early trauma in } \\
\text { adult attachment } \\
\text { and how social } \\
\text { anxiety plays in to } \\
\text { psychosis. }\end{array}$ & $\begin{array}{l}\text { People who } \\
\text { experienced } \\
\text { childhood } \\
\text { trauma and } \\
\text { dysfunctional } \\
\text { parenting seem } \\
\text { to have a higher } \\
\text { instance of } \\
\text { insecure } \\
\text { attachment. }\end{array}$ & $\begin{array}{l}\text { Due to this study being so } \\
\text { specific, there are no other } \\
\text { studies to compare it to, and } \\
\text { there should be more } \\
\text { research in this area. } \\
\text { Additionally, the measures } \\
\text { that were used have can be } \\
\text { subject to bias, so in the } \\
\text { future, another assessment } \\
\text { may be considered. }\end{array}$ \\
\hline 24 & $\begin{array}{c}\text { Richard P. Bentall, } \\
\text { Charles Fernyhough, } \\
2008\end{array}$ & $\begin{array}{l}\text { Environmental } \\
\text { effects on } \\
\text { psychosis }\end{array}$ & $\begin{array}{l}\text { The influence of } \\
\text { environment on the } \\
\text { manifestation of } \\
\text { psychosis. }\end{array}$ & $\begin{array}{l}\text { This article seeks } \\
\text { to remind } \\
\text { practitioners } \\
\text { that integrated } \\
\text { therapies are } \\
\text { likely the most } \\
\text { helpful for } \\
\text { treating } \\
\text { psychosis. }\end{array}$ & $\begin{array}{l}\text { This study lists several } \\
\text { hypotheses that can/should } \\
\text { be examined and tested to } \\
\text { provide further information } \\
\text { on the thesis that body and } \\
\text { mind are connected and } \\
\text { react to trauma from the } \\
\text { genetic material to the } \\
\text { entire person. }\end{array}$ \\
\hline 25 & $\begin{array}{c}\text { Paul H. Lysaker, Petr } \\
\text { Bob, Ondrej Pec, Jay } \\
\text { Hamm, Marina Kukula, } \\
\text { Jen Vohs, Raffaele } \\
\text { Popolo, Giampaolo } \\
\text { Salvatore, Giancarlo } \\
\text { Dimaggio, } 2013\end{array}$ & Metacognition & $\begin{array}{l}\text { The purpose of this } \\
\text { study was to } \\
\text { understand if people } \\
\text { with schizophrenia } \\
\text { engage in synthetic } \\
\text { metacognition, } \\
\text { something that can } \\
\text { effect recovery. }\end{array}$ & $\begin{array}{c}\text { This article } \\
\text { discusses how } \\
\text { deficits in } \\
\text { metacognition } \\
\text { and other brain } \\
\text { processes may } \\
\text { effect persons } \\
\text { with psychotic } \\
\text { disorders, } \\
\text { because it } \\
\text { inhibits their }\end{array}$ & $\begin{array}{l}\text { The article focuses on } \\
\text { metacognition and does not } \\
\text { examine other constructs. } \\
\text { Additionally, it does not } \\
\text { examine if the } \\
\text { metacognitive processes } \\
\text { were absent prior to the first } \\
\text { episode of psychosis. }\end{array}$ \\
\hline
\end{tabular}




\begin{tabular}{|c|c|c|c|c|c|}
\hline & & & & $\begin{array}{l}\text { psychosocial } \\
\text { functioning. }\end{array}$ & \\
\hline 26 & $\begin{array}{c}\text { Lisa J. PHillips, Shona } \\
\text { M. Francey, Jane } \\
\text { Edwards, Nancy } \\
\text { McMurray, } 2009\end{array}$ & Coping & $\begin{array}{l}\text { Literature review } \\
\text { discussing how } \\
\text { people cope with } \\
\text { symptoms } \\
\text { associated with their } \\
\text { psychotic disorder. }\end{array}$ & $\begin{array}{l}\text { Due to life } \\
\text { stressors } \\
\text { increasing the } \\
\text { possibility of a } \\
\text { neurobiological } \\
\text { change that } \\
\text { would bring on } \\
\text { psychotic } \\
\text { symptoms, } \\
\text { focusing on how } \\
\text { people cope } \\
\text { with stress is } \\
\text { important for } \\
\text { treatment. }\end{array}$ & $\begin{array}{l}\text { Some of the studies had } \\
\text { relatively small sample sizes, } \\
\text { or had no comparison } \\
\text { groups. Another limitation } \\
\text { was the lack of longitudinal } \\
\text { research or not discussing } \\
\text { other factors in the study. } \\
\text { Overall, this study was able } \\
\text { to cohesively define several } \\
\text { coping strategies that may } \\
\text { help some patients with a } \\
\text { disorder on the psychotic } \\
\text { spectrum. }\end{array}$ \\
\hline 27 & $\begin{array}{c}\text { Ranil Tan, Rachel V. } \\
\text { Gould, Helen Combes, } \\
\text { Sarah Lehmann, } 2014\end{array}$ & Recovery & $\begin{array}{l}\text { This article seeks to } \\
\text { understand what a } \\
\text { person is going } \\
\text { through in early } \\
\text { psychosis and how } \\
\text { they perceive } \\
\text { barriers to recovery. }\end{array}$ & $\begin{array}{l}\text { This study is } \\
\text { comprehensive } \\
\text { with practitioner } \\
\text { points, urging } \\
\text { the reader to } \\
\text { realize that } \\
\text { there are many } \\
\text { things that must } \\
\text { be considered } \\
\text { when treating } \\
\text { someone } \\
\text { recovering from } \\
\text { psychosis. }\end{array}$ & $\begin{array}{l}\text { The study lists its limitations } \\
\text { as: small homogenous } \\
\text { sample size, and the } \\
\text { program in which they found } \\
\text { their participants means that } \\
\text { those not in this particular } \\
\text { program were included. The } \\
\text { strengths of this study are } \\
\text { that it clearly identifies that } \\
\text { recovery from psychosis is } \\
\text { "multifaceted" and that } \\
\text { including "individual, social } \\
\text { and systemic factors" is } \\
\text { important when discussing } \\
\text { recovery. }\end{array}$ \\
\hline 28 & Kielty Turner (2009) & Mindfulness & $\begin{array}{l}\text { The purpose of this } \\
\text { study was to }\end{array}$ & $\begin{array}{l}\text { Careful } \\
\text { explanation of }\end{array}$ & $\begin{array}{l}\text { Qualitative article that } \\
\text { focuses on the importance }\end{array}$ \\
\hline
\end{tabular}




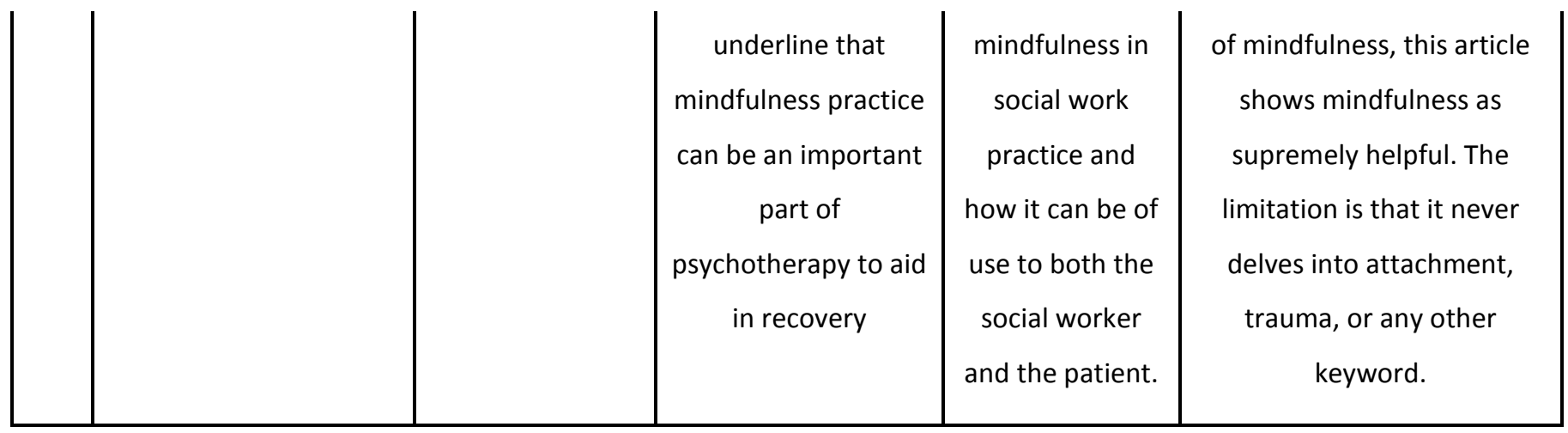

